\title{
Thermodynamical Limit for Correlated Gaussian Random EnERGy Models
}

\author{
P. Contucci, M. Degli Esposti, C. Giardinà, S. Graffi \\ Dipartimento di Matematica
}

Università di Bologna, 40127 Bologna, Italy

e-mail: \{contucci,desposti,giardina,graffi\}@dm.unibo.it

To Francesco Guerra on his sixtieth birthday

\begin{abstract}
Let $\left\{E_{\sigma}(N)\right\}_{\sigma \in \Sigma_{N}}$ be a family of $\left|\Sigma_{N}\right|=2^{N}$ centered unit Gaussian random variables defined by the covariance matrix $C_{N}$ of elements $c_{N}(\sigma, \tau):=\operatorname{Av}\left(E_{\sigma}(N) E_{\tau}(N)\right)$, and $H_{N}(\sigma)=-\sqrt{N} E_{\sigma}(N)$ the corresponding random Hamiltonian. Then the quenched thermodynamical limit exists if, for every decomposition $N=N_{1}+N_{2}$, and all pairs $(\sigma, \tau) \in \Sigma_{N} \times \Sigma_{N}$ :

$$
c_{N}(\sigma, \tau) \leq \frac{N_{1}}{N} c_{N_{1}}\left(\pi_{1}(\sigma), \pi_{1}(\tau)\right)+\frac{N_{2}}{N} c_{N_{2}}\left(\pi_{2}(\sigma), \pi_{2}(\tau)\right)
$$

where $\pi_{k}(\sigma), k=1,2$ are the projections of $\sigma \in \Sigma_{N}$ into $\Sigma_{N_{k}}$. The condition is explicitly verified for the Sherrington-Kirckpatrick, the even p-spin, the Derrida REM and the Derrida-Gardner GREM models.
\end{abstract}

\section{Introduction, Definitions and Results}

It has recently been proved by Guerra and Toninelli GuTO that for the Sherrington-Kirckpatrick (hereafter SK) model (as well as for the even- $p$ spin models) the thermodynamical limit exists for the quenched free energy 
and almost everywhere for its random realizations. In this paper we single out general sufficient conditions that imply the existence of the quenched thermodynamical limit for any correlated Gaussian random energy model. Our analysis thus includes as special cases not only the even $p$ spin models (in particular the SK one, $p=2$ ) but also the Derrida REM model De1, De2] and the Derrida-Gardner GREM DeGa.

The paper is organized as follows: in this section we introduce the definitions and state the results. In section 3, after introducing and elucidating the operation of lifting for a family of Gaussian random variables, we describe the proof of our theorem. In section 4 we show how our analysis applies to the specific examples listed above.

To define the set up we consider a disordered model having $2^{N}$ energy levels where $N$ is the size of the system. We label the energy levels by the index $\sigma=\left\{\sigma_{1}, \sigma_{2}, \ldots, \sigma_{N}\right\}$ where each $\sigma_{i}$ takes the values \pm 1 for $i=1, \ldots, N$. We denote $\Sigma_{N}$ the set of all $\sigma$. Then $\left|\Sigma_{N}\right|=2^{N}$. Clearly $\Sigma_{N}$ coincides with the space of all possible $2^{N}$ Ising configurations of length $N$.

Definition 1 Denote $\left\{E_{\sigma}(N)\right\}_{\sigma \in \Sigma_{N}}$ a family of $2^{N}$ centered unit Gaussian random variables:

$$
\operatorname{Av}\left(E_{\sigma}(N)\right)=0
$$

and covariance matrix $C_{N}$ with elements defined by

$$
\begin{gathered}
c_{N}(\sigma, \sigma):=\operatorname{Av}\left(E_{\sigma}^{2}(N)\right)=1, \\
c_{N}(\sigma, \tau):=\operatorname{Av}\left(E_{\sigma}(N) E_{\tau}(N)\right) .
\end{gathered}
$$

Here $\operatorname{Av}(-)$ denotes expectation with respect to the probability measure

$$
d P\left(E_{1}, \ldots, E_{2^{N}}\right)=\frac{1}{\sqrt{(2 \pi)^{2^{N}} \operatorname{det}(C)}} e^{-\frac{1}{2}\left\langle E, C^{-1} E\right\rangle} d E_{1} \cdots d E_{2^{N}} .
$$




\section{Definition 2}

1. For each $N$ the Hamiltonian is given by

$$
H_{N}(\sigma)=-\sqrt{N} E_{\sigma}(N)
$$

2. The partition function of the system is:

$$
Z_{N}(\beta, E)=\sum_{\sigma} e^{-\beta H_{N}(\sigma)}=\sum_{\sigma} e^{\beta \sqrt{N} E_{\sigma}(N)}
$$

3. The quenched free energy $f_{N}(\beta)$ of the system is defined as:

$$
-\beta f_{N}(\beta):=\alpha_{N}(\beta):=\frac{1}{N} \operatorname{Av}\left(\ln Z_{N}(\beta, E)\right) .
$$

Remark 1 From now on we write $E_{\sigma}(N)=E_{\sigma}$, dropping the $N$-dependence. Remark moreover that Definition 1 includes Gaussian families of the form

$$
\begin{array}{r}
E_{\sigma}(N)=J_{0}+\sum_{i} J_{i} \sigma_{i}+\sum_{i, j} J_{i, j} \sigma_{i} \sigma_{j}+\sum_{i, j, k} J_{i, j, k} \sigma_{i} \sigma_{j} \sigma_{k}+ \\
+\ldots+\sum_{i_{1}, i_{2}, \ldots, i_{N}} J_{i_{1}, i_{2}, \ldots, i_{N}} \sigma_{i_{1}} \sigma_{i_{2}} \ldots \sigma_{i_{N}}
\end{array}
$$

in which every $J$ is an indipendent Gaussian variable.

\section{Examples.}

1. The SK model. Consider first the model defined by

$$
E_{\sigma}:=\frac{1}{N} \sum_{i, j=1}^{N} J_{i, j} \sigma_{i} \sigma_{j}
$$

where the $J_{i, j}$ are $N^{2}$ i.i.d. unit Gaussian random variables. A short computation yields

$$
\operatorname{Av}\left(E_{\sigma} E_{\tau}\right)=\left[q_{N}(\sigma, \tau)\right]^{2}
$$


where, as usual

$$
q_{N}(\sigma, \tau):=\frac{1}{N} \sum_{k=1}^{N} \sigma_{k} \tau_{k}
$$

is the overlap between the $\sigma$ and $\tau$ spin configurations. The standard SK model is instead defined by

$$
E_{\sigma}^{S K}:=\frac{1}{N} \sum_{i<j=1}^{N} J_{i, j} \sigma_{i} \sigma_{j} .
$$

However the quenched free energy densities (7) of the two models coincide up to a rescaling of the temperature, i.e.:

$$
\alpha_{N}^{S K}(\sqrt{2} \beta)=\alpha_{N}(\beta),
$$

In fact, $J_{i, j} \sigma_{i} \sigma_{j}$ are centered, unit and i.i.d. Gaussian random variables $\forall(i, j)$, and $J_{i, j} \sigma_{i} \sigma_{j}=J_{j, i} \sigma_{j} \sigma_{i}$. Hence $J_{i, j} \sigma_{i} \sigma_{j}+J_{j, i} \sigma_{j} \sigma_{i} \stackrel{\mathcal{D}}{=} \sqrt{2} J_{i, j} \sigma_{i} \sigma_{j}$ (here $\stackrel{\mathcal{D}}{=}$ denotes equality in distribution of two random variables). Therefore, taking into account also the $N$ diagonal terms:

$$
\sqrt{N} E_{\sigma} \stackrel{\mathcal{D}}{=} \sqrt{N} \sqrt{2} E_{\sigma}^{S K}+J
$$

where $J$ is a centered unit Gaussian variable. By (6, (6) formula (13) immediately yields the relation (12).

2. The $p$-spin models. Here we consider the model:

$$
E_{\sigma}:=\sqrt{\frac{1}{N^{p}}} \sum_{i_{1}, \ldots, i_{p}=1}^{N} J_{i_{1}, \ldots, i_{p}} \sigma_{i_{1}} \cdots \sigma_{i_{p}}
$$

where the $J_{i_{1}, \ldots, i_{p}}$ are once more i.i.d. unit Gaussian random variables. As before, a short computation yields

$$
\operatorname{Av}\left(\mathrm{E}_{\sigma} \mathrm{E}_{\tau}\right)=\left[q_{N}(\sigma, \tau)\right]^{p}
$$


3. The Derrida REM. Here the model is specified by Definition 1 with

$$
\operatorname{Av}\left(E_{\sigma} E_{\tau}\right)=\delta(\sigma, \tau)
$$

4. The Derrida-Gardner GREM. Its inclusion into the above framework is described in detail in Section 3.3.

Definition 3 For each $\sigma \in \Sigma_{N}$ let $\pi_{1}$ and $\pi_{2}$ be the two canonical projections over the two subsets $\Sigma_{N_{1}}$ and $\Sigma_{N_{2}}$, generated by a partition $\mathcal{P}$ of the coordinates $\left(\sigma_{1}, \ldots, \sigma_{N}\right)$ into a subset of $N_{1}$ coordinates and into a complementary set of $N_{2}$ coordinates: $N_{1}+N_{2}=N, \Sigma_{N}=\Sigma_{N_{1}} \times \Sigma_{N_{2}}, \pi_{1} \otimes \pi_{2}=1_{\Sigma_{N}}$.

(Example: $N=4 ; \sigma \in \Sigma_{4}$ with coordinates denoted $\left\{\sigma_{1}, \sigma_{2}, \sigma_{3}, \sigma_{4}\right\}$. Consider for $N_{1}=N_{2}=2$ the partition $\mathcal{P} \sigma=\left(\sigma_{1}, \sigma_{2}\right) \cup\left(\sigma_{3}, \sigma_{4}\right)$. Then $\Sigma_{N}=\Sigma_{N_{1}} \times \Sigma_{N_{2}}$ and the two projections $\pi_{k}: \Sigma_{N} \rightarrow \Sigma_{N_{k}}, k=1,2$ act in the following way: $\pi_{1}\left(\sigma_{1}, \sigma_{2}, \sigma_{3}, \sigma_{4}\right)=\left(\sigma_{1}, \sigma_{2}\right)$ and $\left.\pi_{2}\left(\sigma_{1}, \sigma_{2}, \sigma_{3}, \sigma_{4}\right)=\left(\sigma_{3}, \sigma_{4}\right)\right)$.

Our main result is the following:

THEOREM 1 Let the covariance matrices $C_{N}$ fulfill the condition:

$$
c_{N}(\sigma, \tau)-\frac{N_{1}}{N} c_{N_{1}}\left(\pi_{1}(\sigma), \pi_{1}(\tau)\right)-\frac{N_{2}}{N} c_{N_{2}}\left(\pi_{2}(\sigma), \pi_{2}(\tau)\right) \leq 0,
$$

for every $N \geq \tilde{N}$, every $(\sigma, \tau) \in \Sigma_{N} \times \Sigma_{N}$ and every decomposition $N_{1}+N_{2}=$ $N$. Then the thermodynamical limit exists, in the sense that

$$
\lim _{N \rightarrow \infty} \frac{1}{N} \operatorname{Av}\left(\log Z_{N}(\beta)\right)=\sup _{N} \frac{1}{N} \operatorname{Av}\left(\log Z_{N}(\beta)\right) .
$$

Remark 2 The result (18) can be extended to the almost-everywhere convergence of free energy density, internal energy and ground state energy with elementary probability methods (see [GuTd]) 
Remark 3 The conditions (17) are not necessary. The proof itself will show that we only need the sign of the quantity in the left hand side of (17) in average, not pointwise. Moreover the condition (11) can be replaced by a more general small deviation vanishing for large $N$ and (2) by a uniform (in $\mathrm{N}$ ) bound over the diagonal terms. We plan to return over such a general case elsewhere.

Remark 4 It is still an open interesting question whether the class of models we control the thermodynamical limit of do have, in that limit, the properties axiomatically introduced by Ruelle in [Ru1 to define directly the infinite particle systems. To this purpose see [BS], BoKu1], BoKu2] and BoKu3].

\section{Proof}

Within this section it is useful to consider 2 identical copies of the same system: the system 1 is assigned the Hamiltonian $H(\sigma)$ and the system 2 the Hamiltonian $H(\tau)$.

Definition 4 The quenched measure over the two copies $\langle-\rangle$ is defined by

$$
<->=\operatorname{Av}[Z(\beta, E)]^{-2} \sum_{(\sigma, \tau) \in \Sigma_{N} \times \Sigma_{N}}-e^{\beta(H(\sigma)+H(\tau))} .
$$

The definition may of course be generalized to $r$ copies.

We want now to embed a Gaussian system $\left\{E_{\sigma}\right\}_{\Sigma_{K}}$ into a larger one $\left\{E_{\tau}\right\}_{\Sigma_{L}}$ for some $K<L$. In particular we want to embed two of them of size $N_{1}$ and $N_{2}$ into one of size $N=N_{1}+N_{2}$. Our embedding procedure is 
defined in terms of the two canonical projections $\pi_{j}, j=1,2$ from $\Sigma_{N}$ to $\Sigma_{N_{j}}$ given in Definition (3).

Definition 5 Given the family $\left\{E_{\mu}\right\}_{\Sigma_{N_{1}}}$ of size $N_{1}$ we lift it to one of size $N:\left\{E_{\sigma}^{(1)}\right\}_{\Sigma_{N}}$ defining

$$
E_{\sigma}^{(1)} \stackrel{\mathcal{D}}{=} E_{\pi_{1}(\sigma)}
$$

Moreover starting from $\left\{E_{\mu}\right\}_{\Sigma_{N_{2}}}$ we define in the same way $\left\{E_{\sigma}^{(2)}\right\}_{\Sigma_{N}}$ by

$$
E_{\sigma}^{(2)} \stackrel{\mathcal{D}}{=} E_{\pi_{2}(\sigma)}
$$

Having defined each family $\left\{E_{\sigma}\right\}_{\Sigma_{N}},\left\{E_{\sigma}^{(1)}\right\}_{\Sigma_{N_{1}}}$ and $\left\{E_{\sigma}^{(2)}\right\}_{\Sigma_{N_{2}}}$ we specify their joint distribution requiring mutual independence.

Remark 5 : The embedded Gaussian systems $\left\{E_{\sigma}^{(1)}\right\}_{\Sigma_{N_{1}}}$ and $\left\{E_{\sigma}^{(2)}\right\}_{\Sigma_{N_{2}}}$ are degenerate: In fact for all $\sigma$ and $\tau$ such that $\pi_{1}(\sigma)=\pi_{1}(\tau)$

$$
E_{\sigma}^{(1)}=E_{\tau}^{(1)}
$$

Summarizing we define the joint measure of $\left\{E_{\sigma}\right\}_{\Sigma_{N}},\left\{E_{\sigma}^{(1)}\right\}_{\Sigma_{N_{1}}}$ and $\left\{E_{\sigma}^{(2)}\right\}_{\Sigma_{N_{2}}}$ $d \hat{P}=d P d P_{1} d P_{2}$ defined by the three covariances $C_{N}, C_{N_{1}}$ and $C_{N_{2}}$.

\section{Proof of THEOREM 1.}

We proceed in three lemmas.

\section{Lemma 0. Interpolation}

Given a pair $\left(\pi_{1}, \pi_{2}\right)$ as before, following [GuTo], we pick three independent Gaussian systems $E_{\pi_{j}(\sigma)}^{(j)}, j=0,1,2$ and introduce the quantity $\left(\pi_{0}(\sigma)=\sigma\right)$

$$
H_{\left(N, N_{1}, N_{2}\right)}(\sigma, t):=-\sum_{j=0}^{2} \sqrt{t_{j} N_{j}} E_{\pi_{j}(\sigma)}^{(j)}
$$


where $t_{0}=t$ and $t_{1}=t_{2}=(1-t)$, and the correspondent partition sum

$$
Z_{N}(t, \beta):=\sum_{\sigma \in \Sigma_{N}} e^{-\beta H_{\left(N, N_{1}, N_{2}\right)}(\sigma, t)} .
$$

It is now easy to see that:

$$
Z_{N}(1, \beta)=Z_{N}(\beta)
$$

and

$$
\begin{aligned}
Z_{N}(0, \beta) & =\sum_{\sigma \in \Sigma_{N}} e^{\beta\left(\sqrt{N_{1}} E_{\pi_{1}(\sigma)}^{(1)}+\sqrt{N_{2}} E_{\pi_{2}(\sigma)}^{(2)}\right)} \\
& =\sum_{\tau \in \Sigma_{N_{2}}} \sum_{\sigma \in \Sigma_{N} ; \pi_{2}(\sigma)=\tau} e^{\beta\left(\sqrt{N_{1}} E_{\pi_{1}(\sigma)}^{(1)}+\sqrt{N_{2}} E_{\tau}^{(2)}\right)} \\
& =\sum_{\tau \in \Sigma_{N_{2}}} e^{\beta \sqrt{N_{2}} E_{\tau}^{(2)}} \sum_{\gamma \in \Sigma_{N_{1}}} e^{\beta \sqrt{N_{1}} E_{\gamma}^{(1)}} \\
& =Z_{N_{1}}(\beta) \cdot Z_{N_{2}}(\beta)
\end{aligned}
$$

\section{Lemma 1. Boundedness}

The Jensen inequality

$$
\operatorname{Av}(\log Z) \leq \log (\operatorname{Av}(Z))
$$

implies

$$
\frac{1}{N} \operatorname{Av}\left(\log Z_{N}(\beta)\right) \leq \log (2)+\frac{\beta^{2}}{2}
$$

because by (6) $\mathrm{Av}(Z)=2 e^{\beta^{2} / 2}$ after performing the Gaussian integration. Lemma 2. Monotonicity

Taking the $t$ derivative of the logarithm of (24) we get: (here we abbreviate $\left.H_{N, N_{1}, N_{2}}=H\right)$

$$
\frac{d}{d t} \log Z_{N}(t)=\frac{\beta}{Z_{N}(t)} \sum_{\sigma \in \Sigma_{N}}\left(\sum_{k=0}^{2} \epsilon_{k} \sqrt{\frac{N_{k}}{t_{k}}} E_{\pi_{k}(\sigma)}^{(k)} e^{-\beta H(\sigma, t)}\right),
$$


where $\epsilon_{0}=1$ and $\epsilon_{1}=\epsilon_{2}=-1$.

We now use the integration by parts formula for correlated Gaussian variables $\left\{\xi_{i}\right\}$ with covariance $c_{i, j}$, which states

$$
\operatorname{Av}\left(\xi_{j} \cdot f\right)=\operatorname{Av}\left(\sum_{k=1}^{n} c_{j, k} \cdot \frac{\partial f}{\partial \xi_{k}}\right)
$$

This yields

$$
\begin{aligned}
& \operatorname{Av}\left(\frac{1}{\beta} \frac{d}{d t} \log Z_{N}(t)\right)=\sum_{\sigma \in \Sigma_{N}} \sum_{k=0}^{2} \epsilon_{k} \sqrt{\frac{N_{k}}{t_{k}}} \operatorname{Av}\left(\frac{E_{\pi_{k}(\sigma)}^{(k)} e^{-\beta H}}{Z_{N}(t)}\right) \\
& =\sum_{\sigma \in \Sigma_{N}} \sum_{k=0}^{2} \epsilon_{k} \sqrt{\frac{N_{k}}{t_{k}}} \operatorname{Av}\left(\sum_{\tau_{k} \in \Sigma_{N_{k}}} c_{N_{k}}\left(\pi_{k}(\sigma), \tau_{k}\right) \cdot \frac{\partial}{\partial E_{\tau_{k}}^{(k)}} \frac{e^{-\beta H}}{Z_{N}(t)}\right)
\end{aligned}
$$

Given now $\tau_{k} \in \Sigma_{N_{k}}$ fixed, we calculate

$$
\begin{aligned}
& \frac{\partial}{\partial E_{\tau_{k}}^{(k)}} \frac{e^{-\beta H(\sigma, t)}}{Z_{N}(t)}=\beta \frac{\sqrt{N_{k} t_{k}} \delta_{\tau_{k}}^{\pi_{k}(\sigma)} e^{-\beta H(\sigma, t)} \cdot Z_{N}(t)-e^{-\beta H(\sigma, t)} \cdot \frac{\partial Z_{N}}{\partial E_{\tau_{k}}^{(k)}}}{Z_{N}^{2}(t)} \\
= & \beta \frac{\sqrt{N_{k} t_{k}} \delta_{\tau_{k}}^{\pi_{k}(\sigma)} e^{-\beta H(\sigma, t)} \cdot Z_{N}(t)-\sqrt{N_{k} t_{k}} e^{-\beta H(\sigma, t)} \cdot \sum_{\xi \in \Sigma_{N}, \pi_{k}(\xi)=\tau_{k}} e^{-\beta H(\xi, t)}}{Z_{N}^{2}(t)}
\end{aligned}
$$

The term with $k=0$ in formula (31) is easy to calculate and we get:

$$
\begin{aligned}
& N \beta \operatorname{Av}\left(\sum_{\sigma \in \Sigma_{N}} \sum_{\tau \in \Sigma_{N}} c_{N}(\sigma, \tau)\left[\delta_{\tau}^{\sigma} \frac{e^{-\beta H(\sigma, t)}}{Z_{N}}-\sum_{\xi \in \Sigma_{N}} \delta_{\xi}^{\tau} e^{-\beta(H(\xi, t)+H(\sigma, t))}\right]\right)= \\
= & N \beta \operatorname{Av}\left(\sum_{\sigma \in \Sigma_{N}} c_{N}(\sigma, \sigma) \cdot \frac{e^{-\beta H(\sigma, t)}}{Z_{N}}-\sum_{(\sigma, \tau) \in \Sigma_{N} \times \Sigma_{N}} c_{N}(\sigma, \tau) e^{-\beta(H(\tau, t)+H(\sigma, t))}\right) \\
= & N \beta\left\langle 1-c_{N}(\sigma, \tau)\right\rangle_{t},
\end{aligned}
$$

where $<->_{t}$ is the quenched measure with respect to the Hamiltonian (23).

In the same way for the term $k=1$ (and similarly for $k=2$ ) we obtain: 


$$
\begin{gathered}
N_{1} \beta \operatorname{Av}\left(\sum_{\sigma \in \Sigma_{N}} \sum_{\tau \in \Sigma_{N_{1}}} c_{N_{1}}\left(\pi_{1}(\sigma), \tau\right)\left[\delta_{\pi_{1}(\sigma)}^{\tau} \frac{e^{-\beta H(\sigma, t)}}{Z_{N}}-\sum_{\xi \in \Sigma_{N}} \delta_{\pi_{1}(\xi)}^{\tau} e^{-\beta(H(\xi, t)+H(\sigma, t))}\right]\right)= \\
=N_{1}\left\langle 1-c_{N_{1}}\left(\pi_{1}(\sigma), \pi_{1}(\tau)\right)\right\rangle_{t} .
\end{gathered}
$$

Summing up the three contributions we obtain:

$$
\begin{gathered}
\frac{1}{N} \frac{d}{d t} \operatorname{Av}\left(\log Z_{N}(t)\right)= \\
=-\beta^{2}<c_{N}(\sigma, \tau)-\frac{N_{1}}{N} c_{N_{1}}\left(\pi_{1}(\sigma), \pi_{1}(\tau)\right)-\frac{N_{2}}{N} c_{N_{2}}\left(\pi_{2}(\sigma), \pi_{2}(\tau)\right)>_{t},
\end{gathered}
$$

and, by the hypothesis (17):

$$
\frac{d}{d t} \operatorname{Av}\left(\log Z_{N}(t)\right) \geq 0 .
$$

Formula (35) together with the boundary conditions (25) and (26) gives for every $N_{1}+N_{2}=N$

$$
\alpha_{N} \geq \frac{N_{1}}{N} \alpha_{N_{1}}+\frac{N_{2}}{N} \alpha_{N_{2}} .
$$

This entails Theorem [1 as explained for instance in [Ru2].

Remark 6 Lemma 3 is indeed a particular case of a theorem by J-P. Kahane [《] (see also [LT], Theorem 3.11, p.74). The Gaussian process $X$ of [ $\mathbb{K}$ can in fact be identified with our Gaussian process $\sqrt{N} E$, and the process $Y$ with our process $\sqrt{N_{1}} E^{(1)}+\sqrt{N_{2}} E^{(2)}$. The further identifications $A \equiv \Sigma_{N} \times \Sigma_{N}$, $B=\emptyset, f \equiv \ln Z$ immediately entail that Hypothesis (1) of [K] reduces to (17) and Assertion (3) to our formula (36) because Hypothesis (2) is just convexity of $\ln Z$. 


\section{$3 \quad$ Examples}

\subsection{The SK and even $p$-spin models}

For the sake of completeness we recover here the Guerra-Toninelli result GuTo. First note that by the definition (10) we have

$$
q_{N}(\sigma, \tau)-\frac{N_{1}}{N} q_{N_{1}}\left(\pi_{1}(\sigma), \pi_{1}(\tau)\right)-\frac{N_{2}}{N} q_{N_{2}}\left(\pi_{2}(\sigma), \pi_{2}(\tau)\right)=0
$$

so that (17) holds as an equality for $p=1$ (the random field model). By (36) this means that the random field model free energy density doesn't depend on the size: $\alpha_{N}=\alpha_{1}$. For $p=2 u$ (SK corresponds to $u=1$ ) formula (37) together with the convexity of the function $x \rightarrow x^{2 u}$ implies (17):

$$
q_{N}^{2 u}(\sigma, \tau)-\frac{N_{1}}{N} q_{N_{1}}^{2 u}\left(\pi_{1}(\sigma), \pi_{1}(\tau)\right)-\frac{N_{2}}{N} q_{N_{2}}^{2 u}\left(\pi_{2}(\sigma), \pi_{2}(\tau)\right) \leq 0
$$

For the standard $p$-spin model defined as

$$
E_{\sigma}=\sqrt{\frac{p !}{2 N^{p}}} \sum_{i_{1}<\ldots<i_{p}} J_{i_{1}, \ldots, i_{p}} \sigma_{i_{1}} \cdots \sigma_{i_{p}}
$$

we refer to GuTo

\subsection{The REM}

The model is defined by:

$$
\operatorname{Av}\left(E_{\sigma} E_{\sigma^{\prime}}\right)=\delta_{\sigma, \sigma^{\prime}}
$$

Condition (17) is verified because it becomes

$$
\delta_{\sigma, \sigma^{\prime}} \leq \frac{N_{1}}{N} \delta_{\pi_{1}(\sigma), \pi_{1}\left(\sigma^{\prime}\right)}+\frac{N_{2}}{N} \delta_{\pi_{2}(\sigma), \pi_{2}\left(s^{\prime}\right)} \cdot
$$


In fact if $\sigma=\sigma^{\prime}$ the previous formula is an identity. If $\sigma \neq \sigma^{\prime}$ the left hand side is 0 but the right hand side is not always zero. Let us take for instance $\sigma=(+,+)$ and $\sigma^{\prime}=(+,-), \pi_{1}(+,+)=+, \pi_{1}(+-)=+, \pi_{2}(+,+)=+$, $\pi_{2}(+,-)=-$. In that case the left hand side is zero and the right hand side is $1 / 2$.

\subsection{The GREM}

To show the inclusion in our scheme of the Derrida-Gardner GREM DeGa let us first recall its construction. The GREM considers $2^{N}$ Gaussian random energies $H(\mu)=\sqrt{N} E_{\mu}$. Their covariance is specified after the assignment of a rooted tree with $n$ layers and $2^{N}$ leaves, $n<N$. The root furcates into $\alpha_{1}^{N}$ branches, the vertices at the end of the first layer furcate into $\alpha_{2}^{N}$ branches etc., up to the vertices at the end of the $n-1$ layer which $\alpha_{n}^{N}$-furcate into the $2^{N}$ leaves.

Remark 7 The topological constraint over the successive furcations which end up on $2^{N}$ leaves implies $\prod_{i=1}^{n} \alpha_{i}^{N}=2^{N}$. Each $\alpha_{i}^{N}$ is an integer which by the previous formula divides $2^{N}$. By the fundamental theorem of arithmetics $\alpha_{i}^{N}=2^{k_{i}}$. Here $k_{i}, i=1, \ldots, n$ is a non-negative integer, and $k_{1}+k_{2}+\ldots k_{n}=$ $N$. In other words: given any tree with $2^{N}$ leaves the construction allows only for furcations in powers of 2 at each layer. The cofficients $\alpha_{i}$ must depend on $N$ : in fact, $\alpha_{i}=2^{\frac{k_{i}}{N}}$ and the only $N$-independent choice of the vector $\alpha$ is obtained for $k_{i}=\frac{N}{l_{i}}$ where the integers $l_{i}$ have to divide $N$ for all $N$. Hence they must fulfill the constraint $\sum_{i=1}^{n} \frac{1}{l_{i}}=1$ which is impossible. 
The previous remark allows us to associate to each leave $\mu$ a spin configuration $\left\{\sigma_{1}, \sigma_{2}, \ldots, \sigma_{N}\right\}$. This can be done observing that the $\alpha_{1}(N)^{N}=2^{k_{1}(N)}$ branches emerging from the root identify canonically the configurations of $k_{1}$ spins, the successive branches the configuration of $k_{2}$ spins and so on. We have in this way associated to each leaf either a path (the only one joining the root to it) or a spin configuration. The model is finally specified by the formula $E(\mu)=\sum_{i=1}^{n} \epsilon_{i}^{(\mu)}$ where the $\epsilon_{i}$ are thrown according to $n$ Gaussians with $\operatorname{Av}\left(\epsilon_{i}\right)=0$ and $\operatorname{Av}\left[\left(\epsilon_{i}\right)^{2}\right]=a_{i}$ : to each branch of the tree we associate an independent $\epsilon$ whose distribution depends (through its variance) only at which layer starts the branch. Defining $v^{(l)}=\sum_{i=1}^{l-1} a_{i}, \quad\left(v^{(0)}=0\right.$ and $\left.v^{(1)}=1\right)$ it is immediate to prove that if two paths $\mu$ and $\nu$ merge at the level $l$ we have $\operatorname{Av}\left(E_{\mu} E_{\mu}\right)=v^{(l)}$. For fixed $n$ and $N$ this construction is exactly the Derrida-Gardner process over a tree $\mathcal{T}_{n, N}$; we will denote it $\left\{\mathcal{E}, \mathcal{T}_{n, N}\right\}$.

Theorem 1 entails existence of the thermodynamical limit for the GREM, in the sense that if $\left\{\mathcal{E}, \mathcal{T}_{n, N}\right\}$ is assigned for a given $n$ and all $N>n$, and the sequence of $\left\{\mathcal{T}_{n, N}\right\}$ is increasing i.e. $k_{i}(N) \geq k_{i}(M)$ for $N \geq M$ then its free energy density is (at fixed n) decreasing (and bounded) in $N$. To show this assertion, starting from a process $\left\{\mathcal{E}, \mathcal{T}_{n, N_{1}}\right\}$ we build the process $\left\{\mathcal{E}_{\pi_{1}}^{(1)}, \mathcal{T}_{n, N}\right\}$ with $N=N_{1}+N_{2}$ in the following way: at each vertex of the tree $\mathcal{T}_{n, N_{1}}$ sitting on the layer $i$ we increase the multiplicity of the furcation by a factor $2^{k_{i}(N)-k_{i}\left(N_{1}\right)}$ assigning the same value $\epsilon_{i}^{(1)}$ to all newly introduced branches. By construction the new process will enjoy the property

$$
\operatorname{Av}\left(E_{\pi_{1}(\sigma)}^{(1)} E_{\pi_{1}(\tau)}^{(1)}\right) \geq v^{(l)}
$$


We apply the same construction to build $\left\{\mathcal{E}_{\pi_{2}}^{(2)}, \mathcal{T}_{n, N}\right\}$ and we have

$$
\operatorname{Av}\left(E_{\pi_{2}(\sigma)}^{(2)} E_{\pi_{2}(\tau)}^{(2)}\right) \geq v^{(l)}
$$

It is now straightforward to verify that conditions (42) and (43) imply (17).

Acknowledgments. One of us (P.C.) thanks Francesco Guerra for useful conversations and Michael Aizenman for introducing him to the Correlated Gaussian Random Energy Models. We also thank the referees and Anton Bovier for interesting observations and for pointing out the reference [K].

This work has been partially supported by the EC RTN-HPRN-CT-2000-00103 (Mathematical Aspects of Quantum Chaos) and by Università di Bologna, Funds for Selected Research Topics.

\section{References}

[BS] E.Bolthausen and A.S.Sznitman, On Ruelle's probability cascades and an abstract cavity method, Commun. Math. Phys. 197 247-276, (1998)

[Bo] A.Bovier, "Statistical Mechanics of Disordered Systems" MaPhySto Lecture Notes 10, Aarhus, 2001.

[BoKu1] A. Bovier, I. Kurkova. Derrida's generalized random energy models. 3. Models with continuous hierarchies, preprint 729, U. Paris 6, 2002

[BoKu2] A. Bovier, I. Kurkova. Derrida's generalized random energy models. 2. Gibbs measures and probability cascades, preprint 728, U Paris 6, 2002

[BoKu3] A. Bovier, I. Kurkova. Derrida's generalized random energy models. 1. Poisson cascades and extremal processes, preprint 727, U. Paris 6, 2002 
[De1] B. Derrida, Random energy model: limit of a family of disordered system, Phys. Rev. Lett. 4579 (1980).

[De2] B. Derrida, Random energy model: an exactly solvable model of disordered system, Phys. Rev. B 242613 (1981).

[DeGa] B.Derrida, E.Gardner: Solution of the generalised random energy model, J.Phys. C 19 2253-2274 (1986)

[GuTo] F. Guerra, F.L. Toninelli: The thermodynamical limit in mean field spin glass model, Comm. Math. Phys. 2002

[K] J.-P. Kahane: Une inegalite du type de Slepian et Gordon sur les processus gaussiens, Isreal J. Math. 55, 109-110 (1985)

[LT] M. Ledoux and M. Talagrand: Probability on Banach spaces. Springer Verlag, (1990)

[Ru1] D. Ruelle: A Mathematical reformualtion of Derrida's REM and GREM, Communication in Mathematical Physics, 108, 225-239, (1987)

[Ru2] D. Ruelle, : Statistical Mechanics. Rigorous results. W.A. Benjamin Inc., New York, (1969) 